\title{
Wavelength-selective ultraviolet (Mg,Zn)O photodiodes: Tuning of parallel composition gradients with oxygen pressure
}

Zhipeng Zhang, Holger von Wenckstern, Jörg Lenzner, and Marius Grundmann

Citation: Appl. Phys. Lett. 108, 243503 (2016); doi: 10.1063/1.4954375

View online: https://doi.org/10.1063/1.4954375

View Table of Contents: http://aip.scitation.org/toc/apl/108/24

Published by the American Institute of Physics

\section{Articles you may be interested in}

Visible-blind and solar-blind ultraviolet photodiodes based on $\left(\mathrm{In}_{\mathrm{x}} \mathrm{Ga}_{1-\mathrm{x}}\right)_{2} \mathrm{O}_{3}$

Applied Physics Letters 108, 123503 (2016); 10.1063/1.4944860

A review of $\mathrm{Ga}_{2} \mathrm{O}_{3}$ materials, processing, and devices

Applied Physics Reviews 5, 011301 (2018); 10.1063/1.5006941

Energy-selective multichannel ultraviolet photodiodes based on $(\mathrm{Mg}, \mathrm{Zn}) \mathrm{O}$

Applied Physics Letters 103, 171111 (2013); 10.1063/1.4826596

Temperature dependent self-compensation in Al- and Ga-doped $\mathrm{Mg}_{0.05} \mathrm{Zn}_{0.95} \mathrm{O}$ thin films grown by pulsed laser deposition

Journal of Applied Physics 120, 205703 (2016); 10.1063/1.4968544

Oxygen vacancies and donor impurities in $\beta-\mathrm{Ga}_{2} \mathrm{O}_{3}$

Applied Physics Letters 97, 142106 (2010); 10.1063/1.3499306

Intrinsic electron mobility limits in $\beta-\mathrm{Ga}_{2} \mathrm{O}_{3}$

Applied Physics Letters 109, 212101 (2016); 10.1063/1.4968550

\section{Conference Proceedings}




\title{
Wavelength-selective ultraviolet (Mg,Zn)O photodiodes: Tuning of parallel composition gradients with oxygen pressure
}

\author{
Zhipeng Zhang, Holger von Wenckstern, Jörg Lenzner, and Marius Grundmann \\ Institut für Experimentelle Physik II, Fakultät für Physik und Geowissenschaften, Universität Leipzig, \\ Linnéstraße 5, 04103 Leipzig, Germany
}

(Received 27 November 2015; accepted 8 June 2016; published online 16 June 2016)

\begin{abstract}
We report on ultraviolet photodiodes with integrated optical filter based on the wurtzite $(\mathrm{Mg}, \mathrm{Zn}) \mathrm{O}$ thin films. Tuning of the bandgap of filter and active layers was realized by employing a continuous composition spread approach relying on the ablation of a single segmented target in pulsed-laser deposition. Filter and active layers of the device were deposited on opposite sides of a sapphire substrate with nearly parallel compositional gradients. Ensure that for each sample position the bandgap of the filter layer blocking the high energy radiation is higher than that of the active layer. Different oxygen pressures during the two depositions runs. The absorption edge is tuned over $360 \mathrm{meV}$ and the spectral bandwidth of photodiodes is typically $100 \mathrm{meV}$ and as low as $50 \mathrm{meV}$. Published by AIP Publishing. [http://dx.doi.org/10.1063/1.4954375]
\end{abstract}

Up to $95 \%$ of the UVA $(315 \leq \lambda \leq 400 \mathrm{~nm})$ radiation reaches the Earth's surface and can damage skin cells in the basal layer of the epidermis and plays a major part in the skin aging and wrinkling. ${ }^{1,2}$ Visible-blind ultraviolet photodiodes (UV PDs) based on wide bandgap semiconductors ${ }^{3-6}$ can efficiently detect this radiation to, e.g., quantify the daily dose that human beings are subjected to. The ternary $\mathrm{Mg}_{x} \mathrm{Zn}_{1-x} \mathrm{O}$ alloy ${ }^{7}$ in wurtzite modification ( $x<0.45$ for $E_{\mathrm{g}}<4.5 \mathrm{eV}$ (Ref. 8)) is a good candidate for detection of the UVA radiation.

A desirable property of PDs is sensitivity within a distinct wavelength range. ${ }^{9}$ Wavelength-selective UV PDs ${ }^{10,11}$ offer the capability to separately analyze various bands with high spectral resolution. A simple approach is to integrate an optical filter layer within the devices, ${ }^{12-18}$ blocks UV photons with energies larger than its bandgap. Further, to achieve monolithic detection in a wide spectral range, a lateral variation of the chemical composition of the $\mathrm{Mg}_{x} \mathrm{Zn}_{1-x} \mathrm{O}$ layers was realized by large-area offset pulsed-laser deposition (PLD) employing a continuous composition spread (CCS) approach $^{19}$ relying on a single segmented target. ${ }^{13-15,20}$ Besides the advantages concerning the device design, combinatorial approaches are typically resource efficient, whereas using the conventional approach a PLD-target had to be prepared for each data point.

In principle, there are three practical ways to achieve different compositional variations within the two layers to be deposited: usage of (a) two different segmented targets ${ }^{12}$ or a single segmented target, but change of (b) background oxygen pressure $p^{21-23}$ or (c) growth temperature $T_{\text {growth }}{ }^{24}$ during the deposition. The approach (a) allows to get free choice of $p$ and $T_{\text {growth }}$ for the individual layers, and the other two approaches require only a single segmented target. However, for (a) two segmented targets are necessary and spectral bandwidth $E_{\text {bandwidth }}$ will be higher than that for other two methods; the main drawback for (c) is that the non-optimized $T_{\text {growth }}$ may result in inferior crystallographic quality of thin films. In this report, as first and most feasible to realize approach we used process (b). It allows optimal $T_{\text {growth }}$ and small but likely nonuniform $E_{\text {bandwidth }}$ to be evaluated, which is attributed to different spatial variations of the two $\mathrm{Mg}$-gradients due to different $p$ used for growth of the filter and active layer. Within this design, the oxygen pressure dependence of the $\mathrm{Mg}$-incorporation into $(\mathrm{Mg}, \mathrm{Zn}) \mathrm{O}$ thin films ${ }^{8,25,26}$ was technically exploited to enable new functionalities in the present case for the realization of wavelength-selective PDs.

Fig. 1(a) shows the schematic layout of the PD based on metal-semiconductor-metal (MSM) structure. The detector is illuminated from the background thus shadowing by the metal electrodes is irrelevant. Fig. 1(b) shows a scanning electron microscope (SEM) image of a mounted MSMstructure connected with gold wires by conductive epoxy resin on two connection pads. The filter layer is deposited first on a 2 in. in diameter double-sided polished $a$-plane sapphire substrate at a lower $p_{2}=0.2 \mu$ bar. After that the substrate is flipped ex situ and subsequently the active layer is deposited at a higher $p_{1}=16 \mu$ bar. Fig. 1(c) shows a crosssectional scanning electron microscope (SEM) image of the two layers from a measuring point at wafer-center, in which the thickness of the filter layer is much smaller than that of the active layer that will be discussed later in Fig. 3(f). Parallel Mg-gradients can be realized by using the same angular starting position for the segmented target and the substrate for the two growth runs (cf. Fig. 1(a)). The segmented target consists of $\mathrm{ZnO}$-segments containing $2 \mathrm{wt}$. \% and $10 \mathrm{wt} . \% \mathrm{MgO}$, respectively. The target and substrate rotated synchronously with a speed of about $8.3 \mathrm{rpm}$. For more details on the PLD-growth, see Refs. 19 and 27.

The chemical and structural properties of both layers are investigated by means of cathodoluminescence (CL), energy dispersive X-ray spectroscopy (EDX), and X-ray diffraction (XRD) measurements at room temperature. In Figs. 2(a) and 2(b), the spatial dependence of maximal CL intensity of the active (filter) layer labeled as $E_{\mathrm{CL} \text {, max. }}^{\text {active (filer) }}$ is shown as energy false color representation across the entire wafer, respectively. The EDX contour plots of $\mathrm{Mg}$-content for active (filter) layer $x^{\text {active (filter) }}$ are depicted in Figs. 2(d) and 2(e), respectively. The CL- and EDX-maps result from an 


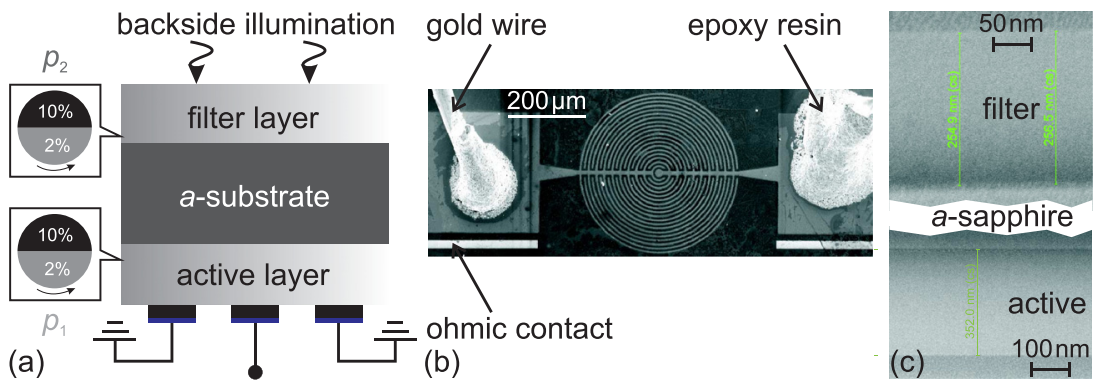

FIG. 1. (a) Schematic layout of a MSM-PD based on CCS (Mg,Zn)O, rotational arrows indicate the synchronized orientation between the target and substrate; (b) SEM images of a mounted MSM-structure and (c) crosssectional SEM image of the two layers.

interpolation from single measured point between neighboring positions visualized by black dots. Obviously, $E_{\text {active (filter) }}$ shifts strictly monotonously with increasing $x^{\text {active(filter) }}$ to higher energies along the white dashed arrows in the direction of Mg-gradients; on the other hand, they are essentially constant along the lines lying in the perpendicular direction. Due to the lower $p_{2}$, a wider range of $E_{\mathrm{CL} \text {, max. }}^{\text {filter }}$ and $x^{\text {filter }}$ is obtained along the Mg-gradients. However, they are not aligned in

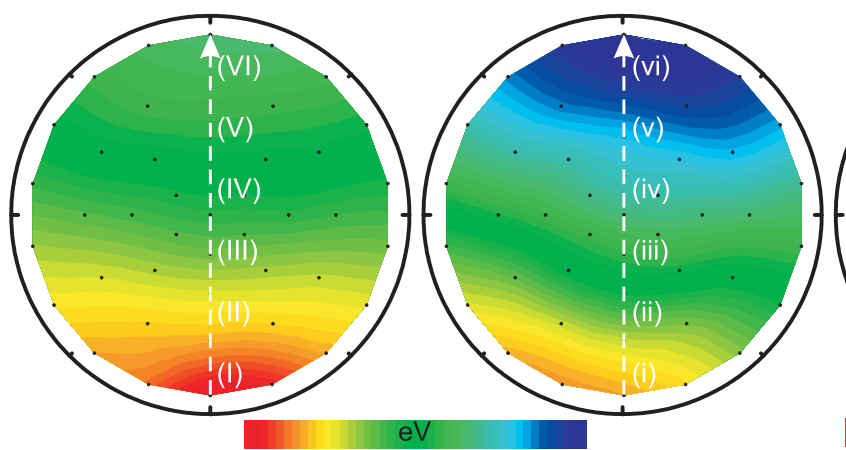

(a)

(a)

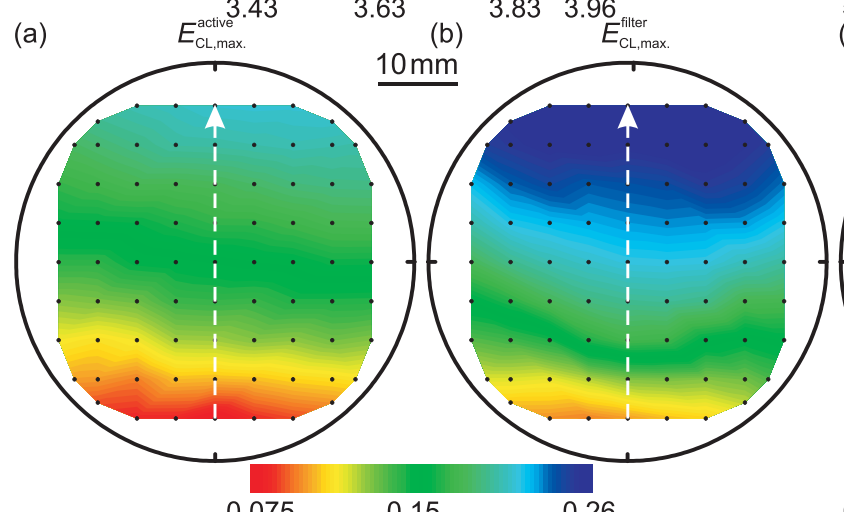

(d)

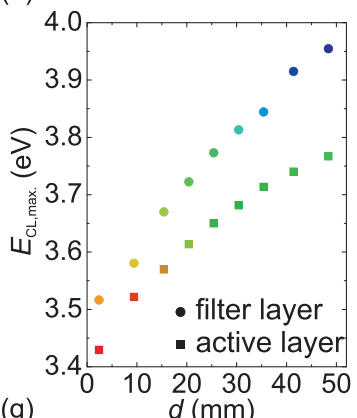

15 (e)

(e) $\quad x^{\text {filter }}$

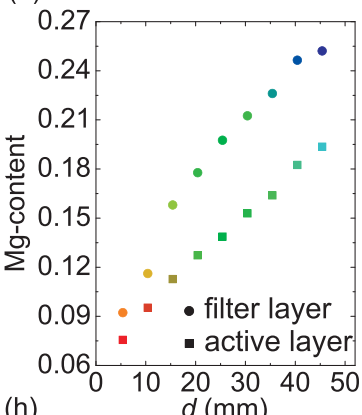

(h) $d(\mathrm{~mm})$

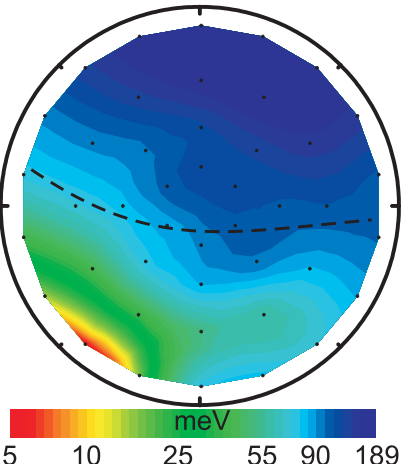

5
(c)

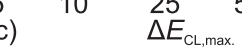

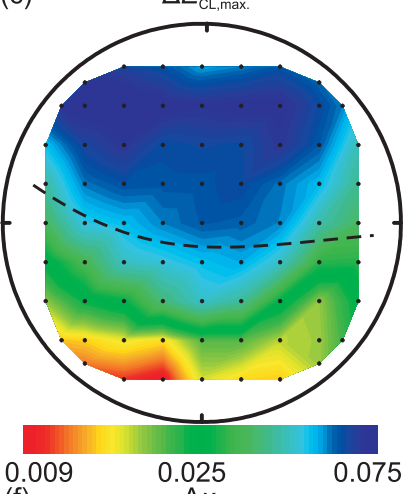

(f)

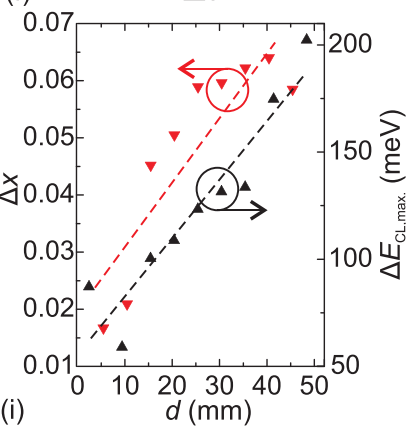

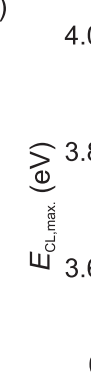

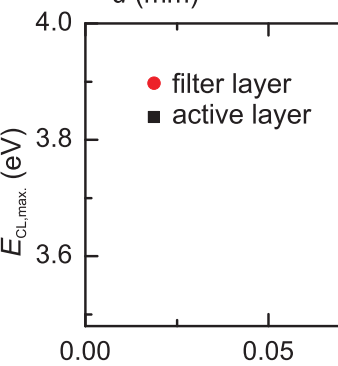

(j)

FIG. 2. False color representations of $E_{\mathrm{CL} \text {,max. }}$ and $x$ of the active (a), (d) and filter (b), (e) layers as well as (c) $\Delta E_{\mathrm{CL} \text {,max. }}$ and (f) $\Delta x$ of the grown sample showing the 2 in. in diameter wafer, respectively; line scans of (g) $E_{C L}^{\text {active (filter) }}$ and (h) $x^{\text {active (filter) }}$ as well as (i) $\Delta x$ and $\Delta E_{\mathrm{CL}, \max }$. versus $d$ at measuring spots along the white dashed arrows; (j) $E_{\mathrm{CL} \text {,max. }}^{\text {active (filter) }}$ versus $x^{\text {active (filter) }}$. 

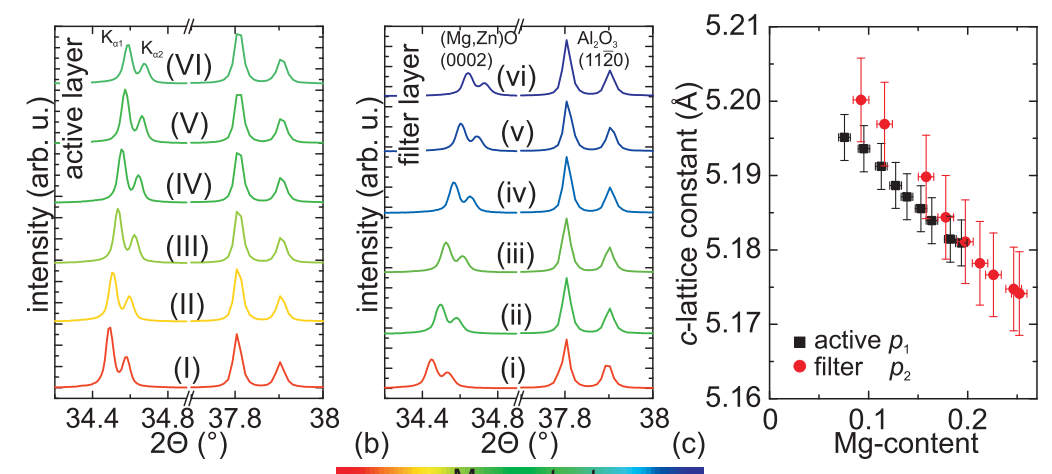

(a)
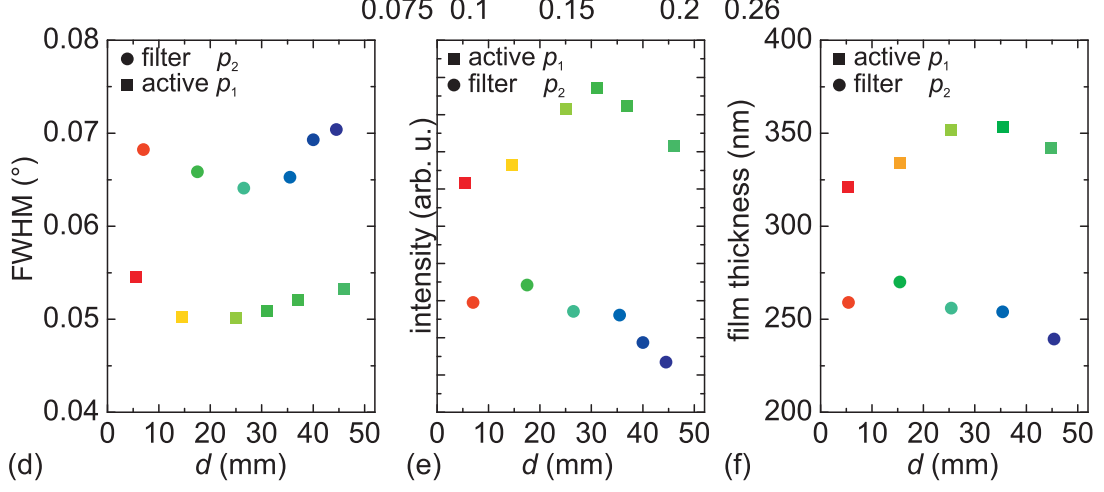

(d)

(e) $d(\mathrm{~mm})$

(f)
FIG. 3. XRD patterns showing $2 \Theta-\omega-$ scans of (a) active and (b) filter layers along the white dashed arrows in Fig. 2, respectively; (c) c-lattice constant vs. $x$; (d) FWHM and (e) peak intensity of (0002) reflections as well as (f) film thickness distribution measured by cross-section SEM in dependence on $d$, respectively; the false color presents various $x$ as same as that in Fig. 2. parallel that is evident in Figs. 2(b) and 2(e) indicative of a slip angle of about $15^{\circ}$ in clockwise direction within the filter layer. A major cause of this technically induced angle is that the mutual position of the segmented target and substrate has not been completely orthogonally adjusted at the beginning of the deposition. To further note, the iso-compositional lines of both layers are not strictly straight. $E_{\mathrm{CL} \text {,max. }}$ and $x$ at the wafer-center are relatively lower as shown in Fig. 2. It is due to a slightly lower $T_{\text {growth }}$ at the wafer-edge for which the $\mathrm{Mg}$ sticking coefficient is higher. ${ }^{13,27}$ This effect is more pronounced for the filter layer since it undergoes a post-growth annealing process during the deposition of the active layer. Both issues will induce a nonuniform $E_{\text {bandwidth }}$ across the wafer.

As expected, since approach (b) was used, the filter and active layer have two different $\mathrm{Mg}$-gradients dependent on the spatial position $d$ as shown in Figs. 2(g)-2(i). Obviously, $E_{\mathrm{CL}, \max \text {. }}^{\text {filer }} x^{\text {filter }}$ have steeper slopes of $9.7 \mathrm{meV} / \mathrm{mm}$ and $4.0 \times 10^{-3} / \mathrm{mm}$ as $E_{\mathrm{CL} \text {, } \operatorname{activ} \text {. }}$ and $x^{\text {active }}$ of $7.0 \mathrm{meV} / \mathrm{mm}$ and $2.9 \times 10^{-3} / \mathrm{mm}$, respectively. This is due to the lower $p_{2}$, which causes a larger degree of inhomogeneity of the expanding plasma plume. ${ }^{19}$ Therefore, a spatial dependent $E_{\text {bandwidth }}$ along the gradient is expected. Figs. 2(c) and 2(f) show the spatial dependence of $\Delta E_{\mathrm{CL} \text {,max. }}=E_{\mathrm{CL} \text {,max. }}^{\text {filter }}-E_{\mathrm{CL} \text {,max. }}^{\text {active }}>0$ and $\Delta x=x^{\text {filter }}-x^{\text {active }}>0$, respectively, indicating that the whole wafer can contribute to the photo response upon backside illumination. The narrowest $E_{\text {bandwidth }}$ could be found at the bottom left corner of the wafer (cf. Fig. 5(c)), where the lowest value of $\Delta E_{\mathrm{CL} \text {,max. }}$ and $\Delta x$ was placed. Fig. 2(j) shows the dependence of $E_{\mathrm{CL} \text {,max. }}$ on $x$ for filter and active layers. Obviously, $E_{\mathrm{CL}, \text { max. }}^{\text {active (filter) }}$ depends linearly on $x^{\text {active (filter) }}$ and exhibits nearly the same slope as $E_{\mathrm{CL}, \max }^{\text {active }}(x) \mathrm{e} \mathrm{V}=(3.28 \pm 0.01) \mathrm{e} \mathrm{V}+$ $(2.50 \pm 0.07) \mathrm{e} \mathrm{V} \cdot x$ and $E_{\mathrm{CL}, \max .}^{\text {filter }}(x) \mathrm{e} \mathrm{V}=(3.26 \pm 0.01) \mathrm{e} \mathrm{V}$ $+(2.62 \pm 0.06) \mathrm{eV} \cdot x$, respectively, which are similar to the results reported in Refs. 7 and 14.
The XRD-patterns of both layers are shown for selected positions along the gradients in Figs. 3(a) and 3(b), respectively. The roman numerals indicated by white dashed arrows in Figs. 2(a) and 2(b) mark these measuring positions. Both layers are single-phase, crystalline, $c$-axis oriented and have wurtzite structure, since, besides the (0002) reflections of wurtzite $\mathrm{Mg}_{x} \mathrm{Zn}_{1-x} \mathrm{O}$, only diffraction peaks connected to the $a$-sapphire substrate of $(11 \overline{2} 0)$ reflection are visible. The (0002) reflections of both layers shift towards higher diffraction angles along the gradients. It indicates that the $c$-lattice constant decreases for increasing $x$ as shown in Fig. 3(c), which is attributed to the slightly lower ionic radii of $\mathrm{Mg}^{2+}$ $(0.57 \AA)$ compared to that of $\left.\mathrm{Zn}^{2+}(0.60 \AA)\right)^{7,8,28}$ The $p$-influence is evident from Fig. 3(c), the lower $p_{2}$ the larger is the $c$-lattice constant (cf. solid red circles). This is attributed to oxygen vacancies in the lattice for lower $p_{2}$. ${ }^{21}$ From XRD patterns for nearly the same $d$, the FWHM/peak intensities of (0002) reflections of the active layer are lower/higher than that of the filter layer, which are depicted in Figs. 3(d) and $3(\mathrm{e})$, respectively. This is attributed to the smaller film thickness of the filter layer grown at the higher $p_{2}{ }^{19,29}$ as shown in Fig. 3(f). This is mainly due to the increased desorption rate of $\mathrm{ZnO}$ and $\mathrm{MgO}$ from the substrate surface for lower $p_{2}$ determined by the conditions of equilibrium nature. ${ }^{8}$

For fabrication of PDs, the whole wafer has been divided into 51 pieces. Each piece has a dimension of about $7 \times 5 \mathrm{~mm}^{2}$ and was fully processed with MSM-structures. After electrical characterization of all MSM-PDs by currentvoltage measurements, the best MSM-structure on each piece with highest Schottky barrier height has been chosen and then mounted on a socket. ${ }^{12,30}$ Basically, this mounted MSM-PD is not representative for the whole sample piece. Within the active area of a single circular MSM-PD of about $\pi(200 \mu \mathrm{m})^{2}$, the variations $\Delta E_{\mathrm{CL} \text {,max. }}^{\text {active }}=2.92(3.80) \mathrm{meV}$. For single piece, this value is estimated to be about $35-50$ 
(50-70) meV. Since the typical dimension of UV PDs for commercial exploitation is about $(10 \mu \mathrm{m})^{2}$, much stronger Mg-gradients are possible for fabrication of MSM-PDs without the loss of spectral resolution, ${ }^{15}$ but larger covered spectral range.

Fig. 4(a) shows the spectral responsivities $\mathcal{R}(\mathrm{A} / \mathrm{W})$ in dependence on incident photon energy $\hbar \omega$ at an external voltage of $V_{\text {ext }}=4.0 \mathrm{~V}$ of 6 MSM-PDs, selected from position a to $\mathrm{f}$ (cf. Fig. 5(c)) along the white dashed arrow in the direction of Mg-gradients (cf. Fig. 2). Please note that measuring spots for the CL- and EDX-characterization as shown in Fig. 2 do not coincide with positions of selected PDs. Low photo responses are measured for $\hbar \omega<E_{\mathrm{g}}^{\text {active }}$ due to the Urbach tail caused by alloy and structural defects. ${ }^{31,32}$ The maximum signal-to-noise ratio of photo response is up to three orders. The combination of both layers would result in a spatial dependence of the absorption edge referred to so-called low $E_{\text {cutoff }}^{\text {low }}$ and high cutoff energies $E_{\text {cutoff }}^{\text {high }}$ defined by $\sqrt{1 / 2} \mathcal{R}_{\text {max. }}$, respectively. Obviously, a blue-shift for both cutoff energies of $\Delta E_{\text {cutoff }}^{\text {low }}=319(348) \mathrm{meV}$ is achieved and depicted in Fig. 4(b) showing the dependence of $E_{\text {cutoff }}^{\text {low (high) }}$ on $x^{\text {active (filter) }}$. The inset of Fig. 4(b) depicts both cutoff energies in dependence on $d$. These results are in good agreement with that of CL-results of $\Delta E_{\text {CL.max. }}^{\text {active filter) }}=340$ (430) meV (cf. Fig. 2(g)). Besides, the responsivities show different slopes as a result of variation of the film thickness of the filter layer depending on $d$ (cf. Fig. 3(f) $)^{13}$ and the inhomogeneity of the Mg-profile along the growth orientation in both layers. ${ }^{14}$ Hence, the device performance upon backside illumination could be improved by further optimization of the fabrication process. Furthermore, for the PD fabricated from position $\mathrm{f}, \mathcal{R}_{\max }$. for
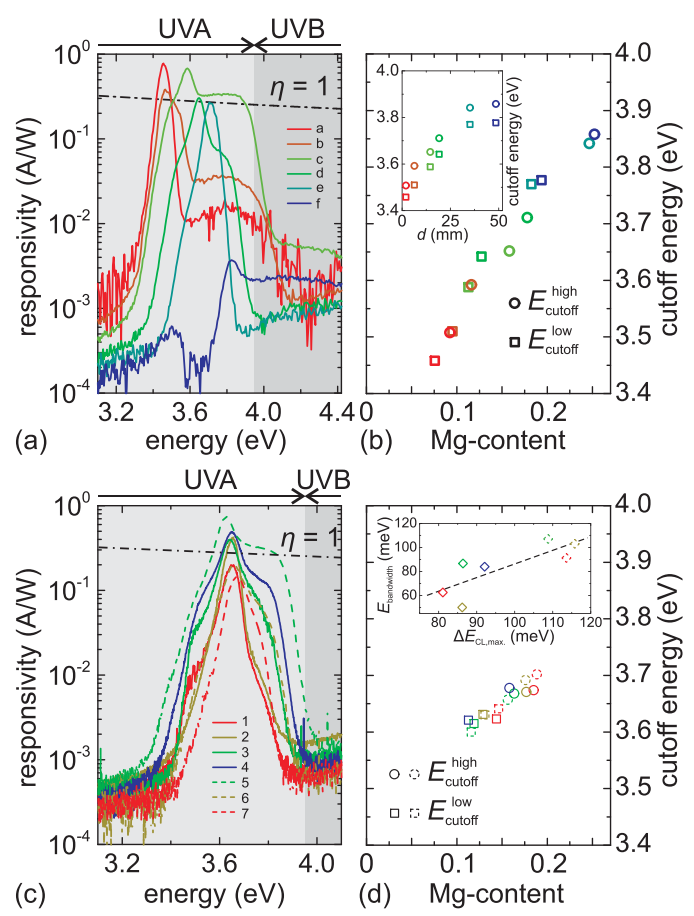

FIG. 4. Spectral responsivity of MSM-PDs along (a) Mg-gradients and (c) a line lying perpendicular to the $\mathrm{Mg}$-gradients indicating as positions from a to $\mathrm{f}$ and 1 to 7 shown in Fig. 5, respectively; (b) and (d) show $E_{\text {cutoff }}^{\text {low (high) }}$ in dependence on $x^{\text {active (filter) }}$ determined from figures (a) and (c), respectively; insets of (b) and (d) show the dependence of $E_{\text {cutoff }}$ and $E_{\text {bandwidth }}$ on $d$ and $\Delta E_{\mathrm{CL}, \max }$ determined from figure (a) and (c), respectively.

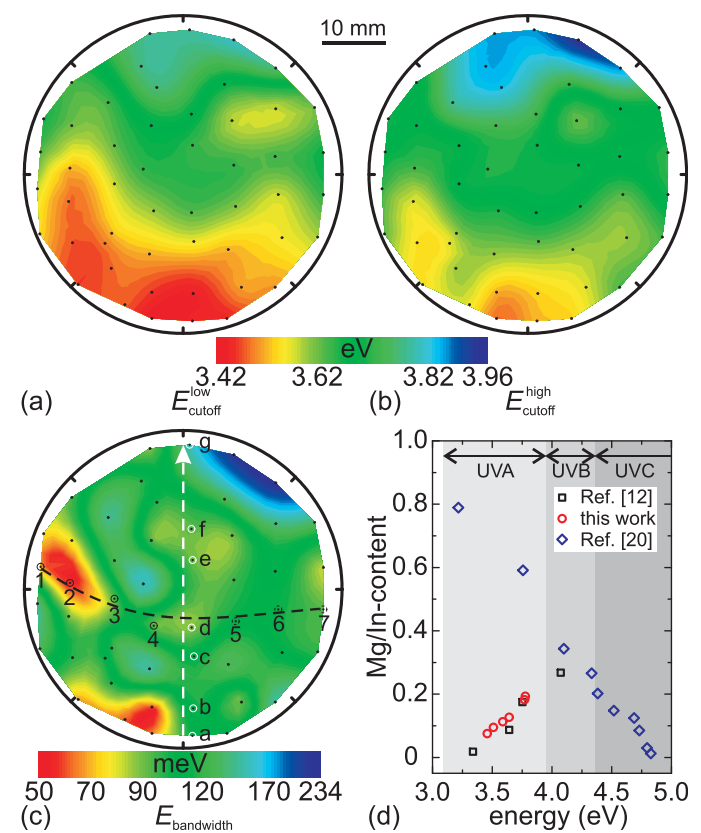

FIG. 5. False color representations of (a) $E_{\text {cutoff }}^{\text {low }}$, (b) $E_{\text {cutoff }}^{\text {high }}$, and (c) $E_{\text {bandwidth }}$, respectively; (d) comparison of $E_{\text {cutoff }}^{\text {low }}$ in dependence on $\mathrm{Mg}$ - and In-content of our UV PDs based on $(\mathrm{Mg}, \mathrm{Zn}) \mathrm{O}$ and $(\mathrm{In}, \mathrm{Ga})_{2} \mathrm{O}_{3}$ thin films, respectively.

$\hbar \omega>E_{\text {cutoff }}^{\text {high }}$ is higher than that for $\hbar \omega<E_{\text {cutoff }}^{\text {low }}$ due to photon absorption and reemission in the filter layer. ${ }^{133}$ Moreover, the maximum responsivities of the devices exceeds the theoretical maximum of external quantum efficiency $\eta=1$ as indicated by a dashed-dotted line in Figs. 4(a) and 4(c). It indicates that the internal gain mechanism exists within the devices, which was explained by trapping of photo-excited holes at metal/(Mg,Zn)O interface. ${ }^{12-14,34}$ Besides, $\mathcal{R}$ as observed in Figs. 4(a) and 4(c) are higher than that of MSM-PDs upon backside illumination reported in Ref. 14, for which the reason remained unclear and further investigations are needed.

Fig. 4(c) shows spectral responsivities of other 7 MSMPDs, but selected from sample stripe along an isocompositional direction (cf. black dashed line in Fig. 2(c)), in which both $\Delta E_{\mathrm{CL} \text {,max. }}$ and $\Delta x$ are nearly the same, respectively. It makes the device fabrications with nearly the same

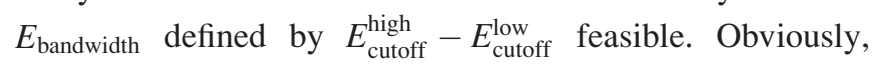
$E_{\text {cutoff }}^{\text {low (high) }}$ of PDs has a red (blue)-shift of photon energy along the black dashed line from position (1) up to (4) in wafer-center, whereas a blue (red)-shift of $E_{\text {cutoff }}^{\text {low (high) }}$ is achieved from position (5) to (7) right along across the wafer. The dependence of $E_{\text {cutoff }}^{\text {low (hig) }}$ on $x^{\text {active (filter) }}$ is shown in Fig. 4(d) having $\Delta E_{\text {cutoff }}^{\text {low (high) }}=41$ (45) meV. This is in agreement with slight variations of $\Delta E_{\mathrm{CL} \text { max. }}^{\text {active (filter) }}=22.6(29.7)$ $\mathrm{meV}$ along the same direction, respectively. Naturally, PDs located on the positions at the wafer-center have a larger $E_{\text {bandwidth }}$ than that from the wafer-edge with $\Delta E_{\text {bandwidth }}=$ $57 \mathrm{meV}$ in this direction. This value is much smaller than $\Delta E_{\text {bandwidth }}=184 \mathrm{meV}$ along the $\mathrm{Mg}$-gradient as depicted in Fig. 5(c). The inset of Fig. 4(d) shows the dependence of $E_{\text {bandwidth }}$ on $\Delta E_{\mathrm{CL} \text {,max. }}$. A linear regression with a slope of 1.13 is obtained indicating a clear correlation between responsivity and bandgap of sample stripe along isocompositional lines. 
The spatial dependence of $E_{\text {cutoff }}^{\text {low }}, E_{\text {cutoff }}^{\text {high }}$, and $E_{\text {bandwidth }}$ of total 51 fabricated MSM-PDs is shown as false color representations in Figs. 5(a)-5(c), respectively. They also result from an interpolation between neighboring positions of corresponding mounted MSM-structure on divided sample pieces marked by black dots. All cutoff energies achieved are in UVA and vary systematically with $\Delta E_{\text {cutoff }}^{\text {low }(\text { igh })}=360(470)$ $\mathrm{meV}$ along the Mg-gradients having the same spatial dependence as expected from CL- and EDX-maps. A wider spectral range corresponding to the filter layer is obtained due to lower $p_{2}$. As expected, $E_{\text {bandwidth }}$ changes systematically along the Mg-gradients from $50 \mathrm{meV}$ (ca. $5 \mathrm{~nm}$ ) up to $234 \mathrm{meV}$ (ca. $20 \mathrm{~nm}$ ). The narrowest and widest $E_{\text {bandwidth }}$ are measured at the position of minimum and maximum $\Delta E_{\mathrm{CL} \text {,max. }}$ and $\Delta x$ (cf. Figs. 2(c) and 2(f)), respectively. As can be seen, only a slight difference of $E_{\text {bandwidth }}$ along the iso-compositional lines is observed and the typical values for most of the PDs is around $100 \mathrm{meV}$ as shown in Fig. 5(c). The unexpected lower $E_{\text {bandwidth }}$ at the left wafer-corner nearby positions 1 and 2 could be explained by local alloy fluctuation within both layers. Therefore, to achieve a uniform narrow $E_{\text {bandwidth }}$, the present approach can be further improved, for which the lower $p_{2}$ for the filter layer should be closer to $p_{1}$ of the active layer.

As mentioned, $(\mathrm{Mg}, \mathrm{Zn}) \mathrm{O}$ in the wurtzite structure is only suitable for UV detection from UVA to UVB $(280 \leq \lambda \leq 315$ $\mathrm{nm})$ spectral range. We have already investigated $(\mathrm{Mg}, \mathrm{Zn}) \mathrm{O}$ layer stacks ${ }^{12}$ as indicated by squares in Fig. 5(c) that are similar to the results from this work as evident as circles. Besides, for solar-blind UV detection we investigated PDs based on group III-sesquioxide ( $\mathrm{In}, \mathrm{Ga})_{2} \mathrm{O}_{3}$ compound $^{20}$ as indicated by diamonds, for which the onset of absorption edge could be tuned from UVC to UVA for decreased In-content.

In summary, we here demonstrated one of three possible ways to realize monolithic, wavelength-selective, visibleblind UV PDs based on the wurtzite $\mathrm{Mg}_{x} \mathrm{Zn}_{1-x} \mathrm{O}$ thin films without dispersive element by the CCS-PLD approach relying on the segmented targets. Two different Mg-gradients of filter and active layer have been deposited at two different background oxygen pressures of 0.2 and $16 \mu \mathrm{bar}$, respectively. The two gradients are almost perfectly aligned in parallel. A blueshift of cutoff energies with $\Delta E_{\text {cutoff }}^{\text {low (high) }}=360$ (470) meV for increasing $x^{\text {active (filter) }}$ along the Mg-gradients is measured, whereas they are nearly constant along iso-compositional lines lying perpendicular to the compositional gradient. Accordingly, an increase of spectral bandwidth from 50 up to $234 \mathrm{meV}$ along the $\mathrm{Mg}$-gradient is observed. The presented results open the way for simultaneous detection and processing of photon signals with designate spectral resolution within a single $2 \mathrm{in}$. in diameter wafer.

This work was supported by Deutsche Forschungsgemeinschaft in the framework of Sonderforschungsbereich 762: "Functionality of Oxide Interfaces." The support of Universität Leipzig within research profile area "Complex Matter" is gratefully acknowledged. The authors are grateful to Mrs. G. Ramm for target preparation, to M. Hahn for sample preparation, to $\mathrm{H}$. Hochmuth and Professor Dr. M. Lorenz for sample growth.
${ }^{1}$ E. Monroy, F. Omnès, and F. Calle, Semicond. Sci. Technol. 18, R33 (2003).

${ }^{2}$ N. S. Agar, G. M. Halliday, R. S. Barnetson, H. N. Ananthaswamy, M. Wheeler, and A. M. Jones, Proc. Natl. Acad. Sci. U.S.A. 101, 4954 (2004).

${ }^{3}$ M. Razeghi and A. Rogalski, J. Appl. Phys. 79, 7433 (1996).

${ }^{4}$ J. Kim, M.-H. Ji, T. Detchprohm, R. D. Dupuis, J.-H. Ryou, A. K. Sood, N. D. Dhar, and J. Lewis, Appl. Phys. Express 8, 122202 (2015).

${ }^{5}$ Q. L. He, Y. H. Lai, Y. Liu, E. Beltjens, J. Qi, and I. K. Sou, Appl. Phys. Lett. 107, 181903 (2015).

${ }^{6}$ H. Chen, H. Liu, Z. Zhang, K. Hu, and X. Fang, Adv. Mater. 28, 403 (2016).

${ }^{7}$ A. Ohtomo, M. Kawasaki, T. Koida, K. Masubuchi, H. Koinuma, Y. Sakurai, Y. Yoshida, T. Yasuda, and Y. Segawa, Appl. Phys. Lett. 72, 2466 (1998).

${ }^{8}$ H. von Wenckstern, R. Schmidt-Grund, C. Bundesmann, A. Müller, C. P. Dietrich, M. Stözel, M. Lange, and M. Grundmann, "The $(\mathrm{Mg}, \mathrm{Zn}) \mathrm{O}$ alloy," in Handbook of Zinc Oxide and Related Materials, edited by Z. C. Feng (CRC Press, Boca Raton, FL, USA, 2012), Chap. 10, p. 257.

${ }^{9}$ S. Pearton, F. Ren, Y.-L. Wang, B. Chu, K. Chen, C. Chang, W. Lim, J. Lin, and D. Norton, Prog. Mater. Sci. 55, 1 (2010).

${ }^{10}$ Y. N. Hou, Z. X. Mei, H. L. Liang, C. Z. Gu, and X. L. Du, Appl. Phys. Lett. 105, 133510 (2014).

${ }^{11}$ D. Jiang, C. Tian, G. Yang, J. Qin, Q. Liang, J. Zhao, J. Hou, and S. Gao, Mater. Res. Bull. 67, 158 (2015).

${ }^{12}$ Z. Zhang, H. von Wenckstern, M. Schmidt, and M. Grundmann, Appl. Phys. Lett. 99, 083502 (2011).

${ }^{13}$ Z. Zhang, H. von Wenckstern, and M. Grundmann, Appl. Phys. Lett. 103, 171111 (2013).

${ }^{14}$ Z. Zhang, H. von Wenckstern, and M. Grundmann, IEEE J. Sel. Top. Quantum Electron. 20, 106 (2014).

${ }^{15} \mathrm{H}$. von Wenckstern, Z. Zhang, J. Lenzner, F. Schmidt, and M. Grundmann, Symposium R - Oxide Semiconductors (Mater. Res. Soc. Symp. Proc., 2014), Vol. 1633, p. 123.

${ }^{16}$ U. Karrer, A. Dobner, O. Ambacher, and M. Stutzmann, J. Vac. Sci. Technol., B 18, 757 (2000).

${ }^{17}$ S. K. Zhang, W. B. Wang, I. Shtau, F. Yun, L. He, H. Morkoç, X. Zhou, M. Tamargo, and R. R. Alfano, Appl. Phys. Lett. 81, 4862 (2002).

${ }^{18}$ M. Brendel, M. Helbling, A. Knauer, S. Einfeldt, A. Knigge, and M. Weyers, Phys. Status Solidi A 212, 1021 (2015).

${ }^{19}$ H. von Wenckstern, Z. Zhang, F. Schmidt, J. Lenzner, H. Hochmuth, and M. Grundmann, CrystEngComm 15, 10020 (2013).

${ }^{20}$ Z. Zhang, H. von Wenckstern, J. Lenzner, M. Lorenz, and M. Grundmann, Appl. Phys. Lett. 108, 123503 (2016).

${ }^{21}$ S. Choopun, R. D. Vispute, W. Noch, A. Balsamo, R. P. Sharma, T. Venkatesan, A. Iliadis, and D. C. Look, Appl. Phys. Lett. 75, 3947 (1999).

${ }^{22}$ M. Lorenz, E. Kaidashev, H. von Wenckstern, V. Riede, C. Bundesmann, D. Spemann, G. Benndorf, H. Hochmuth, A. Rahm, H.-C. Semmelhack, and M. Grundmann, Solid-State Electron. 47, 2205 (2003).

${ }^{23} \mathrm{M}$. Lorenz, in Transparent Conductive Zinc Oxide, "Pulsed Laser Deposition of ZnO-Based Thin Films," edited by K. Ellmer, A. Klein, and B. Rech (Springer, Berlin, Heidelberg, 2008), Chap. 7, p. 303.

${ }^{24}$ S. Choopun, R. D. Vispute, W. Yang, R. P. Sharma, T. Venkatesan, and H. Shen, Appl. Phys. Lett. 80, 1529 (2002).

${ }^{25}$ A. Janotti and C. G. Van de Walle, Phys. Rev. B 75, 121201 (2007).

${ }^{26}$ M. Brandt, H. von Wenckstern, C. Meinecke, T. Butz, H. Hochmuth, M. Lorenz, and M. Grundmann, J. Vac. Sci. Technol., B 27, 1604 (2009).

${ }^{27}$ M. Lorenz, H. Hochmuth, C. Grüner, H. Hilmer, A. Lajn, D. Spemann, M. Brandt, J. Zippel, R. Schmidt-Grund, H. von Wenckstern, and M. Grundmann, Laser Chem. 2010, 140976.

${ }^{28}$ R. D. Shannon, Acta Crystallogr., Sect. A: Found. Crystallogr. 32, 751 (1976).

${ }^{29}$ T. Venkatesan, X. D. Wu, A. Inam, and J. B. Wachtman, Appl. Phys. Lett. 52, 1193 (1988).

${ }^{30}$ A. Lajn, H. v. Wenckstern, Z. Zhang, C. Czekalla, G. Biehne, J. Lenzner, H. Hochmuth, M. Lorenz, M. Grundmann, S. Wickert, C. Vogt, and R. Denecke, J. Vac. Sci. Technol., B 27, 1769 (2009).

${ }^{31}$ M. Grundmann, The Physics of Semiconductors: An Introduction Including Nanophysics and Applications, 2nd ed. (Springer-Verlag, Berlin, Heidelberg, 2010).

${ }^{32}$ J. Huso, H. Che, D. Thapa, A. Canul, M. D. McCluskey, and L. Bergman, J. Appl. Phys. 117, 125702 (2015).

${ }^{33}$ C. Klingshirn, Semiconductor Optics (Springer, Berlin, Heidelberg, 2005).

${ }^{34}$ O. Katz, G. Bahir, and J. Salzman, Appl. Phys. Lett. 84, 4092 (2004). 